\title{
RAPIDLY DECREASING BEHAVIOUR OF SOLUTIONS IN NONLINEAR 3-D-THERMOELASTICITY
}

SONG JIANG

In this paper we study the asymptotic behaviour, as $|x| \rightarrow \infty$, of solutions to the initial value problem in nonlinear three-dimensional thermoelasticity in some weighted Sobolev spaces. We show that under some conditions, solutions decrease fast for each $t$ as $x$ tends to infinity. We also consider the possible extension of the method presented in this paper to the initial boundary value problem in exterior domains.

\section{INTRODUCTION}

We consider the initial value problem (1.1)-(1.4) in $R^{3}$

$$
\begin{gathered}
\partial_{t}^{2} u_{i}-C_{i \alpha j \beta}(\nabla u, \theta) \frac{\partial^{2} u_{j}}{\partial x_{\alpha} \partial x_{\beta}}-\widetilde{C}_{i \alpha}(\nabla u, \theta) \frac{\partial \theta}{\partial x_{\alpha}}=0, \quad i=1,2,3 \\
a(\nabla u, \theta) \partial_{t} \theta-a_{\alpha \beta}(\nabla u, \theta, \nabla \theta) \frac{\partial^{2} \theta}{\partial x_{\alpha} \partial x_{\beta}}-\widetilde{C}_{i \alpha}(\nabla u, \theta) \frac{\partial^{2} u_{i}}{\partial t \partial x_{\alpha}} \\
-b_{i j \alpha}(\nabla u, \theta, \nabla \theta) \frac{\partial^{2} u_{j}}{\partial x_{\alpha} \partial x_{i}}-d_{i}(\nabla u, \theta, \nabla \theta) \frac{\partial \theta}{\partial x_{i}}=0
\end{gathered}
$$

with initial data

$$
u(0)=u^{0}, \quad \partial_{t} u(0)=u^{1}, \quad \theta(0)=\theta^{0},
$$

where repeated indices indicate summation from 1 to $3, u=\left(u_{1}, u_{2}, u_{3}\right)^{T}$ is a vector function, $\theta$ is a scalar function, both depending on $t \in \mathbf{R}_{+}$and $x \in \mathbf{R}^{3}, T$ denotes transposition, $C_{i \alpha j \beta}, \widetilde{C}_{i \alpha}, a, a_{\alpha \beta}, b_{i j \alpha}$ and $d_{i}$ are given smooth functions.

The system (1.1)-(1.3) typically arises in nonlinear thermoelasticity (see Slemrod [9], Racke [8], also see Carlson [2] for extended considerations), where $u$ and $\theta$ stand for the displacement vector and the temperature difference, respectively; and

$$
\begin{aligned}
& C_{i \alpha j \beta}=\frac{\partial^{2} \psi}{\partial\left(\partial u_{j} / \partial x_{\beta}\right) \partial\left(\partial u_{i} / \partial x_{\alpha}\right)}, \quad \tilde{C}_{i \alpha}=\frac{\partial^{2} \psi}{\partial \theta \partial\left(\partial u_{i} / \partial x_{\alpha}\right)}, \quad a=-\frac{\partial^{2} \psi}{\partial \theta^{2}}, \\
& a_{\alpha \beta}=-\frac{\partial q_{\alpha}}{\partial\left(\partial \theta / \partial x_{\beta}\right)} / f(\theta), \quad b_{i j \alpha}=-\frac{\partial q_{i}}{\partial\left(\partial u_{j} / \partial x_{\alpha}\right)} / f(\theta), \quad d_{i}=-\frac{\partial q_{i}}{\partial \theta} / f(\theta),
\end{aligned}
$$

\section{Received 14 February 1990}

This work was supported by the Sonderforschungsbereich 256 of the Deutsche Forschungsgemeinschaft at the University of Bonn.

Copyright Clearance Centre, Inc. Serial-fee code: 0004-9729/91 \$A2.00+0.00. 
$\psi=\psi(\nabla u, \theta)$ is the free Helmholtz energy, $q=q(\nabla u, \theta, \nabla \theta)$ is the heat flux vector and $f(\theta)$ is a $C^{\infty}$ function such that $f(\theta)=\theta+T_{0}$ for $-T_{0} / 2 \leqslant \theta \leqslant T_{0} / 2+\sup _{x}\left|\theta^{0}\right|$ and $0<f_{1} \leqslant f(\theta) \leqslant f_{2}<\infty$ for $-\infty<\theta<\infty, f_{1}, f_{2}$ constants, $T_{0}>0$ the reference temperature.

Note that in the original equations $f(\theta) \equiv \theta+T_{0}$. Here, in order to get rid of the possibly singular term $\theta+T_{0}$, we introduce the transformation $f(\theta)$ (see Slemrod [9]). which is identical to $\theta+T_{0}$ for any non-negative $\theta^{0}$ and small time interval by the local existence, or for small initial data by the global existence stated in Section 3.

Our purpose in this paper is to investigate the rapidly decreasing behaviour of solutions of (1.1)-(1.4) in some weighted Sobolev spaces. The motivation for studying the rapidly decreasing behaviour of solutions of (1.1)-(1.4) is not only the interest in the asymptotic behaviour as $|x| \rightarrow \infty$ itself, but also its importance for numerical solution of (1.1)-(1.4) (see Jiang [5] on utilising the far field behaviour of solutions to solve numerically (1.1)-(1.4) in the one-dimensional case). Recently, the author [4] studied the far field behaviour of solutions to (1.1)-(1.4) in the one-dimensional case by applying the Fourier transform to the related linearised problem. Here, we use a different method to show that solutions of (1.1)-(1.4) decrease fast for each $t$ as $x$ tends to infinity. The main results in this paper are the following.

THEOREM 4.1. Let the conditions of Theorem 3.1 be satisfied. Also assume that $u^{0} \in H_{r}^{3}, u^{1}, \theta^{0} \in H_{r}^{2}$ for $r \geqslant 0$. Then the local solution $u, \theta$ on $\left[0, T^{*}\right]$ of (1.1)-(1.4) established in Theorem 3.1 satisfies

$$
\bar{D}^{2} \bar{D}_{x}^{1} u, \partial_{t} \theta, \bar{D}_{z}^{2} \theta \in L^{\infty}\left(\left[0, T^{*}\right], S_{r}^{s-2}\right)
$$

and

$$
||\left|\bar{D}^{2} \bar{D}_{z}^{1} u(t)\right|\left\|_{r, \rho-2}+\left|\left\|\partial_{t} \theta(t)\left|\left\|_{r, e-2}+\right\|\right|\left|\bar{D}_{z}^{2} \theta(t)\right|\right\|_{r, a-2} \leqslant C E_{r}\right.\right.
$$

for any $t \in\left[0, T^{*}\right]$, where $C=C\left(r, T^{*}, e_{0}\right), e_{0}=\left\|u^{0}\right\|_{0+1,2}+\left\|u^{1}\right\|_{\Omega, 2}+\left\|\theta^{0}\right\|_{\ell, 2}$ and $E_{r}=e_{0}+\left|u^{0}\right|_{r, 3}+\left|u^{1}\right|_{r, 2}+\left|\theta^{0}\right|_{r, 2}$.

ThEOREM 4.2. Let the conditions of Theorem 3.2 be satisfied. Also assume that $u^{0} \in H_{r}^{3}, u^{1}, \theta^{0} \in H_{r}^{2}$ for $r \geqslant 0$. Then for any $T>0$ the global solution $u, \theta$ of (1.1)-(1.4) established in Theorem 3.2 satisfies

$$
\bar{D}^{2} \bar{D}_{x}^{1} u, \quad \partial_{t} \theta, \quad \bar{D}_{z}^{2} \theta \in L^{\infty}\left([0, T], S_{r}^{z-2}\right)
$$

and

$$
\left\|\left|\left\|\overline { D } ^ { 2 } \overline { D } _ { x } ^ { 1 } u ( t ) \left|\left\|_{r, s-2}+\left|\left\|\partial _ { t } \theta ( t ) \left|\left\|_{r, s-2}+\left|\left\|\bar{D}_{x}^{2} \theta(t) \mid\right\|_{r, s-2} \leqslant C(r, s, \delta, T) E_{r}\right.\right.\right.\right.\right.\right.\right.\right.\right.\right.
$$

for any $t \in[0, T]$, where $E_{r}$ is the same as in Theorem 4.1. 
REMARK 1.1.. The notations appearing above are defined in Section 2.

REMARK 1.2. Theorems 4.1 and 4.2 together with the Sobolev imbedding theorem imply that $\left|\bar{D}_{x}^{1} u(t)\right|,\left|\partial_{t} u(t)\right|,|\theta(t)|=O\left(|x|^{-r}\right)$ for each $t$ as $|x| \rightarrow \infty$. Also see Remark 2.1 in Section 2.

It follows from Theorem 4.1, Remark 3.1 and Lemma 2.3 that

Corollary 4.3. Let the conditions of Theorem 3.1 be satisfied. Assume that $u^{0}, u^{1}, \theta^{0} \in S\left(R^{3}\right)$. Then for the local solution $u, \theta$ on $\left[0, T^{*}\right]$, we have

$$
u, \quad \theta \in C^{\infty}\left(\left[0, T^{*}\right], S\left(\mathrm{R}^{3}\right)\right)
$$

The paper is organised as follows: Section 2 gives the notation, the assumptions and proves some lemmas. In Section 3 we state the local, and global existence theorems. In Section 4 we prove Theorems 4.1 and 4.2 , and at the end of the section we consider the extension of the method given in Section 4 to the initial boundary value problem in exterior domains.

\section{ASSUMptions, NOTATIONS AND PRELIMINARIES}

We denote by $W^{m, p}(1 \leqslant p \leqslant \infty, 0 \leqslant m<\infty)$ the usual Sobolev spaces on $\mathbf{R}^{3}$ with the norm $\|\cdot\|_{m, p}$ (see Adams $[1]$ ). $W^{\infty, p}=\cap_{m \in N \cup\{0\}} W^{m, p} .\|\cdot\|$ and $(\cdot, \cdot)$ stand for the norm and the inner product in $L^{2}\left(R^{3}\right)$, respectively. We also use

$$
\partial_{j}=\partial / \partial x_{j}, \quad \partial_{x}^{\alpha}=\partial_{1}^{\alpha_{1}} \partial_{2}^{\alpha_{2}} \partial_{3}^{\alpha_{3}} \quad\left(|\alpha|=\alpha_{1}+\alpha_{2}+\alpha_{3}\right) .
$$

For any integer $L \geqslant 0$,

$$
\begin{array}{ll}
D_{x}^{L} u=\left(\partial_{x}^{\alpha} u ;|\alpha|=L\right), & D^{L} u=\left(\partial_{t}^{j} \partial_{x}^{\alpha} u ; j+|\alpha|=L\right), \\
\bar{D}_{x}^{L} u=\left(\partial_{x}^{\alpha} u ;|\alpha| \leqslant L\right), & \bar{D}^{L} u=\left(\partial_{t}^{j} \partial_{x}^{\alpha} u ; j+|\alpha| \leqslant L\right) .
\end{array}
$$

For a vector valued function $f=\left(f_{1}, \cdots, f_{m}\right)^{T}$ and a non-negative integer $L$ we set

$$
\begin{array}{ll}
D_{z}^{L} f=\left(D_{z}^{L} f_{1}, \cdots, D_{x}^{L} f_{m}\right)^{T}, & D^{L} f=\left(D^{L} f_{1}, \cdots, D^{L} f_{m}\right)^{T}, \\
\bar{D}_{x}^{L} f=\left(\bar{D}_{x}^{L} f_{1}, \cdots, \bar{D}_{z}^{L} f_{m}\right)^{T}, & \bar{D}^{L} f=\left(\bar{D}^{L} f_{1}, \cdots, \bar{D}^{L} f_{m}\right)^{T}
\end{array}
$$

and $f \in X$ (a normed space with norm $\|\cdot\|_{X}$ ) means that each component of $f$ is in $X$ and $\|f\|_{X}=\left\|f_{1}\right\|_{X}+\cdots+\left\|f_{m}\right\|_{X} \cdot S\left(R^{3}\right)$ is the Schwartz space of all rapidly decreasing infinitely differentiable functions on $R^{3}$. Throughout $\sigma(x)=\left(1+|x|^{2}\right)^{1 / 2}$, 
$x \in \mathbf{R}^{3}$. For $r, s \in \mathbf{R}^{1}$ we denote by $H_{r}^{*}$ the completion of $S\left(R^{3}\right)$ in the norm $|u|_{r, 8}=$ $\left\|\sigma^{r} \mathcal{F}^{-1}\left(\sigma^{\alpha} \mathcal{F}(u)\right)\right\|$, where $\mathcal{F}$ and $\mathcal{F}^{-1}$ are the Fourier and inverse Fourier transforms, respectively. For $r \in R^{1}$ and $s \in N \cup\{0\}$, we denote by $W_{r}^{*}$ the completion of $S\left(R^{3}\right)$ in the norm $|u|_{r, \Omega}^{*}=\left(\sum_{|\alpha| \leqslant s}\left\|\sigma^{r} \partial_{x}^{\alpha} u\right\|^{2}\right)^{1 / 2}$. Both $H_{r}^{s}$ and $W_{r}^{s}$ are Hilbert spaces with the inner products $(u, v)_{r, s}=\left(\sigma^{r} \mathcal{F}^{-1}\left(\sigma^{\wedge} \mathcal{F}(u)\right), \sigma^{r} \mathcal{F}^{-1}\left(\sigma^{\wedge} \mathcal{F}(v)\right)\right)$ and $(u, v)_{r, a}^{*}=$ $\sum_{|\alpha| \leqslant \varepsilon}\left(\sigma^{r} \partial_{z}^{\alpha} u, \sigma^{r} \partial_{x}^{\alpha} v\right)$, respectively. $H_{0}^{\prime}$ and $W_{0}^{s}$ coincide with the usual Sobolev spaces $H^{*}$ and $W^{*} \cdot C_{0}^{\infty}\left(\mathrm{R}^{3}\right)$ is the space of all infinitely differentiable functions with compact supports. Let $m \in N \cup\{0\}, X$ be a Banach space with norm $\|\cdot\|_{X}, G \in \mathbf{R}^{1}$ a domain, thus $C^{m}(G, X)$ denotes the space of all $X$-valued functions which are $m$ times continuously differentiable in $G$.

We also introduce the Hilbert space $S_{r}^{:}=S_{r}^{*}\left(\mathrm{R}^{3}\right)$ defined by

$$
S_{r}^{*}=H_{r}^{0} \cap H_{0}^{*}
$$

with the inner product and norm

$$
\begin{aligned}
& ((u, v))_{r, \Delta}=(u, v)_{r, 0}+(u, v)_{0, \Delta}, \\
& \mid\|u, v\|_{r, \Delta}=((u, u))_{r, \Delta}^{1 / 2}=\left(|u|_{r, 0}^{2}+|u|_{0, \Delta}^{2}\right)^{1 / 2} .
\end{aligned}
$$

Throughout this paper, $C$ (sometimes used as $C(a, b, \ldots)$ to emphasise that $C$ depend on $a, b, \cdots)$ will denote various constants in various places.

Now, we state the assumptions for (1.1)-(1.4).

ASSUMPTION 2.1 .

(1) $\psi$ and $q$ are sufficiently smooth functions.

(2) $a_{\alpha \beta}(P, \mu, \nu)=a_{\beta \alpha}(P, \mu, \nu), \quad P \in \mathbf{R}^{3 \times 3}, \mu \in \mathbf{R}^{1}, \nu \in \mathbf{R}^{3}$.

(3) There is a constant $\kappa_{0}>0$ such that

$$
\begin{array}{r}
C_{i \alpha j \beta}(P, \mu) \xi_{i} \xi_{j} \eta_{\alpha} \eta_{\beta} \geqslant \kappa_{0}|\xi|^{2}|\eta|^{2}, \quad a_{\alpha \beta}(P, \mu, \nu) \xi_{\alpha} \xi_{\beta} \geqslant \kappa_{0}|\xi|^{2}, \quad a(P, \mu) \geqslant \kappa_{0} \\
\text { for } P \in \mathbf{R}^{3 \times 3}, \mu \in \mathbf{R}^{1} \text {, and } \nu, \xi=\left(\xi_{1}, \xi_{2}, \xi_{3}\right)^{T}, \eta=\left(\eta_{1}, \eta_{2}, \eta_{3}\right)^{T} \in \mathbf{R}^{3} .
\end{array}
$$

In order to obtain global smooth solutions of (1.1)-(1.4), we also require ASSUMPTION 2.2 .

$$
\begin{aligned}
& \left|C_{i \alpha j \beta}(\nabla u, \theta)-C_{i \alpha j \beta}(0,0)\right|, \quad\left|\tilde{C}_{i \alpha}(\nabla u, \theta)-\tilde{C}_{i \alpha}(0,0)\right|, \\
& |a(\nabla u, \theta)-a(0,0)|=O\left(|\nabla u|^{2}+|\theta|^{2}\right) \quad \text { and } \\
& \left|\frac{\partial q_{\alpha}(\nabla u, \theta, \nabla \theta)}{\partial\left(\partial \theta / \partial x_{\beta}\right)}-\frac{\partial q_{\alpha}(0,0,0)}{\partial\left(\partial \theta / \partial x_{\beta}\right)}\right|, \quad\left|\frac{\partial q_{i}}{\partial\left(\partial u_{j} / \partial x_{\alpha}\right)}\right|,\left|\frac{\partial q_{i}}{\partial \theta}\right|, \\
& \quad=O\left(|\nabla u|^{2}+|\theta|^{2}+|\nabla \theta|^{2}\right)
\end{aligned}
$$


near the origin.

Before proving Theorems 4.1 and 4.2, we need some lemmas. The following two lemmas can be found in Triebel $[10,11]$.

Lemma 2.1. For $s \in N \cup\{0\}, H_{r}^{*}=W_{r}^{*} \cdot|\cdot|_{r, s}$ and $|\cdot|_{r, s}^{*}$ are equivalent norms.

Lemma 2.2. For any $s, r \in R^{1}$,

$$
\left(H_{r}^{s}\right)^{\prime}=H_{-r}^{-s},
$$

where $X^{\prime}$ denotes the dual of a Banach space $X$.

The following lemmas 2.3-2.5 are given in Tsutsumi [12].

Lemma 2.3. $S_{r}^{s} \subset S_{r^{\prime}}^{\prime}$ if $r \geqslant r^{\prime}$ and $s \geqslant s^{\prime}$. The intersection $\cap_{r, a>0} S_{r}^{s}$ $\left(=\cap_{r, \Delta \in N} S_{r}^{a}\left(R^{3}\right)\right)$, equipped with the initial topology, is the Schwartz space $S\left(R^{3}\right)$. Moreover the intersection $\cap_{0} S_{\text {; }}^{*}$ is $S\left(\mathbf{R}^{3}\right)$.

Lemma 2.4. We have

$$
\left(S_{r}^{s}\right)^{\prime}=H_{-r}^{0}+H_{0}^{-s} .
$$

Lemma 2.5. Let $r, s \geqslant 0$. Assume $u \in S_{r}^{r s}\left(\mathbf{R}^{3}\right)$. Then $u \in H_{r-1}^{:}$and

$$
|u|_{r-1, s} \leqslant C(r, s)|| u \mid \|_{r, r s}
$$

Remark 2.1. By Lemma 2.1, 2.5 and the Sobolev imbedding theorem we see that if $u \in S_{r}^{r(s+2)}$ with $s \in N \cup\{0\}$ and $r \in N$, then

$$
\sup _{x \in R^{3}}\left|\sigma^{r-1}(x) \bar{D}_{z}^{s} u(x)\right| \leqslant C(r, s)|u|_{r-1, o+2} \leqslant C(r, s)\left|\|u \mid\|_{r, r(\bullet+2)},\right.
$$

that is $\left|\bar{D}_{x}^{\prime} u(x)\right|=O\left(|x|^{-(r-1)}\right)$ as $|x| \rightarrow \infty$.

The following lemma follows from a straightforward calculation and we shall omit its proof here.

LEMMA 2.6. Let $\rho \in C_{0}^{\infty}\left(\mathrm{R}^{3}\right)$ such that $\rho=1$ if $|x| \leqslant 1$ and $\rho=0$ if $|x| \geqslant 2$. Let $\rho_{\varepsilon}(x)=\rho(\varepsilon x)$ for $0<\varepsilon<1$. Then as $\varepsilon \rightarrow 0$,

$$
\begin{aligned}
& \rho_{\varepsilon}(x) \rightarrow 1 \text { uniformly on any bounded set in } \mathbf{R}^{3}, \\
& D_{x}^{\alpha} \rho_{\varepsilon}(x) \rightarrow 0 \quad \text { uniformly in } \mathbf{R}^{3}, \quad|\alpha| \neq 0 .
\end{aligned}
$$

Moreover, for any $|\alpha| \in N \cup\{0\}$, we have

$$
\left|D_{x}^{\alpha} \rho_{\varepsilon}(x)\right| \leqslant C_{\alpha} \varepsilon^{\gamma}(\sigma(x))^{-(|\alpha|-\gamma)}, \quad 0 \leqslant \gamma \leqslant|\alpha|
$$

where the constant $C_{\alpha}>0$ is independent of $\varepsilon$. 


\section{Local, And Global Existence Theorems}

In this section we give the local and global existence theorems, which are useful in the proofs of Theorems 4.1 and 4.2 in the subsequent section.

Theorem 3.1. (Local existence) Let Assumption 2.1 be satisfied. Let $s \geqslant 5$ be an integer. Assume that $u^{0} \in W^{*+1,2}, u^{1}, \theta^{0} \in W^{*, 2}$. Then there is a $T^{*}>0$ such that (1.1)-(1.4) has a unique solution $u, \theta$ with

$$
\left\{\begin{array}{l}
u \in \cap_{i=0}^{2} C^{i}\left(\left[0, T^{*}\right], W^{\bullet+1-i, 2}\right), \quad \theta \in \cap_{i=0}^{1} C^{i}\left(\left[0, T^{*}\right], W^{s-2 i, 2}\right), \\
\nabla \theta \in L^{2}\left(\left[0, T^{*}\right], W^{*, 2}\right) .
\end{array}\right.
$$

Furthermore, we have

$$
\begin{gathered}
\sum_{i=0}^{2}\left\|\partial_{t}^{i} u(t)\right\|_{\odot+1-i, 2}+\sum_{i=0}^{1}\left\|\partial_{t}^{i} \theta(t)\right\|_{:-2 i, 2}+\left(\int_{0}^{t}\|\nabla \theta(\tau)\|_{e, 2}^{2} d \tau\right)^{1 / 2} \\
\leqslant C\left(\left\|u^{0}\right\|_{-+2,2}+\left\|u^{1}\right\|_{s, 2}+\left\|\theta^{0}\right\|_{s, 2}\right) \quad \text { for any } t \in\left[0, T^{*}\right]
\end{gathered}
$$

where $C>0$ is a constant depending only on $T^{*},\left\|u^{0}\right\|_{\bullet+1,2},\left\|u^{1}\right\|_{\bullet, 2}$ and $\left\|\theta^{0}\right\|_{\&, 2}$.

Proof: See Kawashima [7], Vol'pert and Hudjaev [13], also see Racke [8].

REMARK 3.1. From Theorem 3.1, it follows that if $u^{0}, u^{1}, \theta^{0} \in W^{\infty, 2}$, then $u$, $\theta \in C^{\infty}\left(\left[0, T^{*}\right], W^{\infty, 2}\right)$.

The following global existence theorem was proven by Racke [8].

Theorem 3.2. (Global existence) Let Assumptions 2.1 and 2.2 be satisfied. Let $U^{0}=\left(\nabla u^{0}, u^{1}, \theta^{0}\right)^{T}$. Then there are integers $s, s_{1}, \cdots, s_{6}$ with $s \geqslant 5$ and a small $\delta>0$ such that if $u^{0} \in L^{2}\left(R^{3}\right)$ and

$$
\begin{aligned}
& U^{0} \in W^{*, 2} \cap W^{* 3,9 / 7} \cap W^{* 4,13 / 11} \cap W^{* 0,26 / 15} \cap W^{* 0,18 / 11} \text { with } \\
& \left\|U^{0}\right\|_{8,2}+\left\|U^{0}\right\|_{8_{2}, 9 / 7}+\left\|U^{0}\right\|_{8_{4}, 13 / 11}+\left\|U^{0}\right\|_{8,26 / 15}+\left\|U^{0}\right\|_{8_{8,18 / 11}}<\delta \text {, }
\end{aligned}
$$

there exists a unique smooth solution of (1.1)-(1.4) satisfying

$$
\begin{gathered}
u \in \cap_{i=0}^{2} C^{i}\left([0, \infty), W^{\lrcorner+1-i, 2}\right), \quad \theta \in \cap_{i=0}^{1} C^{i}\left([0, \infty), W^{\lrcorner-2 i, 2}\right), \\
\left\|\left(\nabla u(t), \partial_{t} u(t), \theta(t)\right)^{T}\right\|_{\iota, 2} \leqslant C(s, \delta)\left\|U^{0}\right\|_{\iota, 2}, \quad \forall t \in[0, \infty)
\end{gathered}
$$

\section{The Proof of Theorem 4.1 ANd 4.2}

Proof of Theorem 4.1: By the definition of $S_{r}^{*}\left(R^{3}\right)$ and Theorem 3.1, it suffices to show that

$$
\bar{D}^{2} \bar{D}_{z}^{1} u, \quad \bar{D}_{z}^{2} \theta, \quad \partial_{t} \theta \in L^{\infty}\left(\left[0, T^{*}\right], H_{r}^{0}\right), \quad \nabla \theta \in L^{2}\left(\left[0, T^{*}\right], H_{r}^{2}\right),
$$


and

$$
\left|\left(\bar{D}^{2} \bar{D}_{z}^{1} u, \bar{D}_{z}^{2} \theta, \partial_{t} \theta\right)^{T}(t)\right|_{r, 0}+\left(\int_{0}^{t}|\nabla \theta(\tau)|_{r, 2}^{2} d \tau\right)^{1 / 2} \leqslant C\left(r, T^{*}, e_{0}\right) E_{r} .
$$

for any $0 \leqslant t \leqslant T^{*}$, where $e_{0}$ and $E_{r}$ are the same as in Theorem 4.1.

We shall show (4.1)-(4.2) by induction on $r$. It can be easily seen by Theorem 3.1 that (4.1)-(4.2) are valid for $r=0$. Suppose that (4.1)-(4.2) hold for all values less than or equal to $r-1(r \geqslant 1)$, we show (4.1)-(4.2) for $r$.

Let $\rho(x) \in C_{0}^{\infty}\left(R^{3}\right)$ with $\rho(x)=1$ for $|x| \leqslant 1$ and $\rho(x)=0$ for $|x| \geqslant 2$. Set $\rho_{\varepsilon}(x)=\rho(\varepsilon x)$ for $0<\varepsilon<1$. Denote

$$
u^{e}(t, \cdot)=\rho_{\varepsilon}(\cdot) u(t, \cdot), \quad \theta^{e}(t, \cdot)=\rho_{e}(\cdot) \theta(t, \cdot), \quad t \in\left[0, T^{*}\right],
$$

Now, multiplication of (1.1)-(1.2) with $\rho_{e}$ implies that $u^{e}$ and $\theta^{e}$ satisfy

$$
\begin{gathered}
\partial_{t}^{2} u_{i}^{e}-C_{i \alpha j \beta}(\nabla u, \theta) \frac{\partial^{2} u_{j}^{e}}{\partial x_{\alpha} \partial x_{\beta}}-\widetilde{C}_{i \alpha}(\nabla u, \theta) \frac{\partial \theta^{e}}{\partial x_{\alpha}}=f_{i}^{e}(u, \theta), \\
a(\nabla u, \theta) \partial_{t} \theta^{e}-a_{\alpha \beta}(\nabla u, \theta, \nabla \theta) \frac{\partial^{2} \theta^{e}}{\partial x_{\alpha} \partial x_{\beta}}-\widetilde{C}_{i \alpha}(\nabla u, \theta) \frac{\partial^{2} u_{i}^{e}}{\partial t \partial x_{\alpha}} \\
-b_{i j \alpha}(\nabla u, \theta, \nabla \theta) \frac{\partial^{2} u_{j}^{e}}{\partial x_{\alpha} \partial x_{i}}-d_{i}(\nabla u, \theta, \nabla \theta) \frac{\partial \theta^{e}}{\partial x_{i}}=g^{e}(u, \theta),
\end{gathered}
$$

where

$$
f_{i}^{e}(u, \theta)=-C_{i \alpha j \beta} \frac{\partial^{2} \rho_{\varepsilon}}{\partial x_{\alpha} \partial x_{\beta}} u_{j}-C_{i \alpha j \beta}\left(\frac{\partial \rho_{\varepsilon}}{\partial x_{\alpha}} \frac{\partial u_{j}}{\partial x_{\beta}}+\frac{\partial \rho_{\varepsilon}}{\partial x_{\beta}} \frac{\partial u_{j}}{\partial x_{\alpha}}\right)-\widetilde{C}_{i \alpha} \frac{\partial \rho_{\varepsilon}}{\partial x_{\alpha}} \theta
$$

$$
\begin{aligned}
g^{e}(u, \theta)= & -a_{\alpha \beta} \frac{\partial^{2} \rho_{\varepsilon}}{\partial x_{\alpha} \partial x_{\beta}} \theta-2 a_{\alpha \beta} \frac{\partial \rho_{e}}{\partial x_{\alpha}} \frac{\partial \theta}{\partial x_{\beta}}-\widetilde{C}_{i \alpha} \frac{\partial \rho_{\varepsilon}}{\partial x_{\alpha}} \partial_{t} u_{i} \\
& -b_{i j \alpha} \frac{\partial^{2} \rho_{\varepsilon}}{\partial x_{\alpha} \partial x_{i}} u_{j}-b_{i j \alpha}\left(\frac{\partial \rho_{\varepsilon}}{\partial x_{\alpha}} \frac{\partial u_{j}}{\partial x_{i}}+\frac{\partial \rho_{\epsilon}}{\partial x_{i}} \frac{\partial u_{j}}{\partial x_{\alpha}}\right)-d_{i} \frac{\partial \rho_{\epsilon}}{\partial x_{i}} \theta
\end{aligned}
$$

If we utilise (3) of Assumption 2.1, Lemma 2.1, the energy estimate (3.2) and the Sobolev imbedding theorem, we infer that for $0 \leqslant t \leqslant T^{*}$

$$
\begin{gathered}
\left(\sigma^{2 r} C_{i \alpha j \beta} \frac{\partial u_{i}^{e}}{\partial x_{\alpha}}, \frac{\partial u_{j}^{e}}{\partial x_{\beta}}\right)(t)=\left(C_{i \alpha j \beta} \frac{\partial\left(\sigma^{r} u_{i}^{e}\right)}{\partial x_{\alpha}}, \frac{\partial\left(\sigma^{r} u_{j}^{e}\right)}{\partial x_{\beta}}\right)(t) \\
-\left(C_{i \alpha j \beta} \frac{\partial \sigma^{r}}{\partial x_{\alpha}} u_{i}^{e}, \frac{\partial \sigma^{r}}{\partial x_{\beta}} u_{j}^{e}+\sigma^{r} \frac{\partial u_{j}^{e}}{\partial x_{\beta}}\right)(t)-\left(C_{i \alpha j \beta} \sigma^{r} \frac{\partial u_{i}^{e}}{\partial x_{\alpha}}, \frac{\partial \sigma^{r}}{\partial x_{\beta}} u_{j}^{e}\right)(t) \\
\geqslant c_{0}\left\|\sigma^{r} u^{e}(t)\right\|_{1,2}^{2}-c_{1}\left\|\sigma^{r} u^{e}(t)\right\|^{2}-C\left(r, e_{0}\right)|u(t)|_{r-1,0}^{2}-\left(c_{0} / 2\right)\left|u^{\varepsilon}(t)\right|_{r, 1}^{2},
\end{gathered}
$$


where $c_{0}$ and $c_{1}$ are positive constants. By applying the induction hypotheses and the equality: $f(t)=f(0)+\int_{0}^{t} f^{\prime}(\tau) d \tau$, we arrive at

$$
\begin{gathered}
\left(\sigma^{2 r} C_{i \alpha j \beta} \frac{\partial u_{i}^{e}}{\partial x_{\alpha}}, \frac{\partial u_{j}^{e}}{\partial x_{\beta}}\right)(t) \geqslant \frac{c_{0}}{2}\left|u^{\varepsilon}(t)\right|_{r, 1}^{2} \\
-C\left(r, T^{*}, e_{0}\right)\left\{\left(E_{r}\right)^{2}+\left(E_{r}^{e}\right)^{2}+\int_{0}^{t}\left(\left|u^{e}\right|_{r, 0}^{2}+\left|\partial_{t} u^{e}\right|_{r, 0}^{2}\right)(\tau) d \tau\right\}
\end{gathered}
$$

for any $t \in\left[0, T^{*}\right]$, where

$$
E_{r}^{e}=\left\|\rho_{\varepsilon} u^{0}\right\|_{\triangle+1,2}+\left\|\rho_{\varepsilon} u^{1}\right\|_{s, 2}+\left\|\rho_{\varepsilon} \theta^{0}\right\|_{\rho, 2}+\left|\rho_{\varepsilon} u^{0}\right|_{r, 3}+\left|\rho_{\varepsilon} u^{1}\right|_{r, 2}+\left|\rho_{\varepsilon} \theta^{0}\right|_{r, 2} .
$$

Multiplying (4.4) and (4.5) by $\sigma^{2 r} \partial_{t} u_{i}^{e}$ and $\sigma^{2 r} \theta^{e}$ in $L^{2}\left(R^{3}\right)$, respectively, we have by partial integration and (4.9) that

$$
\begin{gathered}
\left|\partial_{t} u^{e}(t)\right|_{r, 0}^{2}+\left|u^{e}(t)\right|_{r, 1}^{2}+\left|\theta^{e}(t)\right|_{r, 0}^{2}+\int_{0}^{t}\left|\nabla \theta^{e}(\tau)\right|_{r, 0}^{2} d \tau \\
\leqslant C\left(r, T^{*}, e_{0}\right)\left\{\left(E_{r}\right)^{2}+\left(E_{r}^{e}\right)^{2}+\int_{0}^{t}\left(\left|\partial_{t} u^{e}\right|_{r, 0}^{2}+\left|u^{e}\right|_{r, 1}^{2}+\left|\theta^{e}\right|_{r, 0}^{2}\right)(\tau) d \tau\right. \\
\left.+\int_{0}^{t}\left(\left|f^{e}(u, \theta)\right|_{r, 0}^{2}+\left|g^{e}(u, \theta)\right|_{r, 0}^{2}\right)(\tau) d \tau\right\}
\end{gathered}
$$

where $f^{\epsilon}=\left(f_{1}^{\varepsilon}, f_{2}^{\varepsilon}, f_{3}^{\varepsilon}\right)^{T}$ and we have used the estimate:

$$
\begin{gathered}
\sup _{x \in \mathbf{R}^{3}}\left\{\left|\partial_{t} a\right|,\left|\nabla C_{i \alpha j \beta}\right|,\left|\nabla \tilde{C}_{i \alpha}\right|,\left|\nabla a_{\alpha \beta}\right|,\left|\nabla b_{i j \alpha}\right|\right\}(t) \\
\leqslant C\left(e_{0}\right)\left(\|\nabla u(t)\|_{\iota, 2}+\|\theta(t)\|_{,, 2}\right) \leqslant C\left(e_{0}\right) \quad \text { for any } t \in\left[0, T^{*}\right],
\end{gathered}
$$

which follows from (3.2) in Theorem 3.1 and the Sobolev imbedding theorem. Recalling the definitions of $f_{i}^{e}$ and $g^{\varepsilon}$, we apply Lemma 2.6 and the induction hypotheses to deduce

$$
\begin{aligned}
\left|f^{e}(u, \theta)(t)\right|_{r, 0}^{2}, \quad\left|g^{e}(u, \theta)(t)\right|_{r, 0}^{2} & \leqslant C\left(|u(t)|_{r-1,1}^{2}+\left|\partial_{t} u(t)\right|_{r-1,0}^{2}+|\theta(t)|_{r-1,1}^{2}\right) \\
& \leqslant C\left(E_{r}\right)^{2}, \quad \forall t \in\left[0, T^{*}\right] .
\end{aligned}
$$

Inserting (4.13) into (4.11), one gets

$$
\begin{gathered}
\left|\partial_{t} u^{e}(t)\right|_{r, 0}^{2}+\left|u^{e}(t)\right|_{r, 1}^{2}+\left|\theta^{e}(t)\right|_{r, 0}^{2}+\int_{0}^{t}\left|\nabla \theta^{e}(\tau)\right|_{r, 0}^{2} d \tau \\
\leqslant C\left(r, T^{*}, e_{0}\right)\left\{\left(E_{r}\right)^{2}+\left(E_{r}^{e}\right)^{2}+\int_{0}^{t}\left(\left|\partial_{t} u^{\epsilon}\right|_{r, 0}^{2}+\left|u^{e}\right|_{r, 1}^{2}+\left|\theta^{e}\right|_{r, 0}^{2}\right)(\tau) d \tau\right\}
\end{gathered}
$$


for all $0 \leqslant t \leqslant T^{*}$. So, we obtained a bound for $\partial_{t} u^{\varepsilon}, \bar{D}_{z}^{1} u^{\varepsilon}$ and $\theta^{\varepsilon}$. To estimate the derivatives of higher order of $u^{e}$ and $\theta^{\varepsilon}$, we take $\nabla$ and $\nabla a^{-1}$ (respectively $D_{x}^{2}$ and $D_{x}^{2} a^{-1}$ ) on both sides of (4.4) and (4.5), respectively, and multiply with $\sigma^{2 r} \partial_{t} \nabla u_{i}^{\varepsilon}$ and $a \sigma^{2 r} \nabla \theta^{e}$ (respectively with $\sigma^{2 r} \partial_{t} D_{x}^{2} u_{i}^{e}$ and $a \sigma^{2 r} D_{x}^{2} \theta^{e}$ ) in $L^{2}\left(R^{3}\right)$. By arguments similar to those used for (4.14), we obtain

$$
\begin{gathered}
\left|\partial_{t} \nabla u^{e}(t)\right|_{r, 1}^{2}+\left|\nabla u^{e}(t)\right|_{r, 2}^{2}+\left|\nabla \theta^{e}(t)\right|_{r, 1}^{2}+\int_{0}^{t}\left|D_{x}^{2} \theta^{e}(\tau)\right|_{r, 1}^{2} d \tau \\
\leqslant C\left(r, T^{*}, e_{0}\right)\left\{\left(E_{r}\right)^{2}+\left(E_{r}^{e}\right)^{2}+\int_{0}^{t}\left(\left|\partial_{t} \nabla u^{e}\right|_{r, 1}^{2}+\left|\nabla u^{\varepsilon}\right|_{r, 2}^{2}+\left|\nabla \theta^{e}\right|_{r, 1}^{2}\right)(\tau) d \tau\right\} .
\end{gathered}
$$

An application of the Gronwall ineqality to (4.14)-(4.15) gives

$$
\left|\partial_{t} u^{e}(t)\right|_{r, 2}^{2}+\left|u^{e}(t)\right|_{r, 3}^{2}+\left|\theta^{e}(t)\right|_{r, 2}^{2}+\int_{0}^{t}\left|\nabla \theta^{e}(\tau)\right|_{r, 2}^{2} d \tau \leqslant C\left\{\left(E_{r}\right)^{2}+\left(E_{r}^{e}\right)^{2}\right\}
$$

for any $t \in\left[0, T^{*}\right]$, where $C=C\left(r, T^{*}, e_{0}\right)$ is independent of $\varepsilon$. It follows from the equations (4.4)-(4.5), (4.16) and the induction hypotheses that for $t \in\left[0, T^{*}\right]$

$$
\left|\partial_{t}^{2} u^{e}(t)\right|_{r, 1}^{2}+\left|\partial_{t} \theta^{e}(t)\right|_{r, 0}^{2} \leqslant C\left(r, T^{*}, e_{0}\right)\left\{\left(E_{r}\right)^{2}+\left(E_{\tau}^{e}\right)^{2}\right\} .
$$

Thus, $\left\{\bar{D}^{2} \bar{D}_{x}^{1} u^{e}\right\},\left\{\partial_{t} \theta^{e}\right\}$ and $\left\{\bar{D}_{x}^{2} \theta^{e}\right\}$ (respectively $\left\{\nabla \theta^{e}\right\}$ ) remain in a bounded set of $L^{\infty}\left(\left[0, T^{*}\right], H_{r}^{0}\right)$ (respectively $\left.L^{2}\left(\left[0, T^{*}\right], H_{r}^{2}\right)\right)$ provided that $\varepsilon$ is sufficiently small. Hence we can construct subsequences of $\left\{u^{e}\right\}$ and $\left\{\theta^{e}\right\}$, still denoted by $\left\{u^{e}\right\}$ and $\left\{\theta^{e}\right\}$, such that (note here Lemma 2.2 and the fact: $\left(u^{e}, \theta^{e}\right)^{T} \rightarrow(u, \theta)^{T}$ strongly in $L^{\infty}\left([0, T], L^{2}\left(\mathrm{R}^{3}\right)\right)$ as $\left.\varepsilon \rightarrow 0\right)$

$$
\begin{array}{r}
\left(\bar{D}^{2} \bar{D}_{x}^{1} u^{\varepsilon}, \partial_{t} \theta^{e}, \bar{D}_{x}^{2} \theta^{\varepsilon}\right)^{T} \longrightarrow\left(\bar{D}^{2} \bar{D}_{x}^{1} u, \partial_{t} \theta, \bar{D}_{x}^{2} \theta\right)^{T} \in L^{\infty}\left(\left[0, T^{*}\right], H_{r}^{0}\right) \\
\left(\text { weak-*) in } L^{\infty}\left(\left[0, T^{*}\right], H_{r}^{0}\right) \text { as } \varepsilon \rightarrow 0,\right. \\
\nabla \theta^{e} \longrightarrow \nabla \theta \in L^{2}\left(\left[0, T^{*}\right], H_{r}^{2}\right) \text { weakly in } L^{2}\left(\left[0, T^{*}\right], B_{r}^{2}\right) \text { as } \varepsilon \rightarrow 0,
\end{array}
$$

which proves (4.1). The estimate (4.2) immediately follows from the lower semicontinuity properties of the weak-* and weak topologies, and (4.16)-(4.17). Hence (4.1)-(4.2) are valid for $r$. This completes the proof.

Proof of Theorem 4.2: For any $T>0$, we know from Theorem 3.2 that $(u, \theta)^{T}$ satisfies the equations (1.1)-(1.4) on $[0, T]$. Utilising the estimate (3.4), following the same arguments as in the proof of Theorem 4.1 (see the proof of (4.1)-(4.2)), we can show the theorem. 
We close this section by considering the initial boundary value problem (1.1)-(1.4) in an exterior domain $\Omega$ with the Dirichlet boundary conditions

$$
\left.u\right|_{8 n}=0,\left.\quad \theta\right|_{8 n}=0
$$

where $\partial \Omega$ is the boundary of $\Omega$ and sufficiently smooth. For the initial boundary value problem (1.1)-(1.4), (4.19), we have a local existence theorem similar to Theorem 3.1 (see Jiang and Racke [6], Chrzȩszczyk [3], and Zheng and Shen [14]). We may apply the arguments similar to those used for Theorem 4.1 to show that the local solution on $\left[0, T^{*}\right]$ of (1.1)-(1.4) and (4.19) satisfies

$$
\cdot \bar{D}^{2} u, \quad \partial_{t} \theta, \quad \bar{D}_{z}^{2} \theta \in L^{\infty}\left(\left[0, T^{*}\right], W_{r}^{0}(\Omega)\right),
$$

where $W_{r}^{0}(\Omega)=\left\{u \in L^{2}(\Omega) ;|u|_{r, 0, \Omega}=\left(\int_{\Omega}\left|\sigma^{r}(x) u(x)\right|^{2} d x\right)^{1 / 2}<\infty\right\} . W_{r}^{0}(\Omega)$ is a Hilbert space with the inner product $(u, v)_{r, 0, \Omega}=\int_{\Omega} \sigma^{2 r}(x) u(x) \bar{v}(x) d x$. It is worth noticing that a slightly different technique in the derivation of (4.20) is that instead of the differentiations with respect to $x$ as in the derivation of (4.15), we perform the differentiation with respect to $t$ to obtain an estimate for

$$
\left|\left(\partial_{t}^{2} u^{e}, \partial_{t} \nabla u^{e}, \partial_{t} \theta^{e}\right)^{T}(t)\right|_{r, 0, \Omega}^{2}+\int_{0}^{t}\left|\partial_{t} \nabla \theta^{e}(\tau) d \tau\right|_{r, 0, \Omega}^{2} d \tau
$$

similar to (4.15), which together with the equations (4.4), (4.5), (4.19) and the regularity for elliptic systems yields a bound for $\left|\bar{D}_{z}^{2} u^{e}(t)\right|_{r, 0, \Omega}^{2}+\left|\bar{D}_{z}^{2} \theta^{e}(t)\right|_{r, 0, \Omega}^{2}$.

\section{REFERENCES}

[1] R.A. Adams, Sobolev Spaces (Academic Press, New York, 1975).

[2] D.E. Carlson, 'Linear Thermoelasticity', in Handbuch der Physik VIa/2, pp. 297-345 (Springer-Verlag, Berlin, Heidelberg, New York, 1972).

[3] A. Chrzȩszczyk, 'Some existence results in dynamical thermoelasticity; part I nonlinear case', Arch. Mech. 39 (1987), 605-617.

[4] S. Jiang, 'Far field behavior of solutions to the equations of nonlinear 1-d-thermoelasticity', Appl. Anal. 36 (1990), 25-35.

[5] S. Jiang, 'Numerical solution for the Cauchy problem in nonlinear 1-d-thermoelasticity', Computing 44 (1990), 147-158..

[6] S. Jiang and R. Racke, 'On some quasilinear hyperbolic-parabolic initial boundary value problems', Math. Methods Appl. Sci. 12 (1990), 315-319.

[7] S. Kawashima, Systems of a hyperbolic-parabolic composite type, with applications to the equations of magnetohydrodynamics, Thesis (Kyoto University, 1983).

[8] R. Racke, 'On the Cauchy problem in nonlinear 3-d-thermoelasticity', Math. Z. 203 (1990), 649-682. 
[9] M. Slemrod, 'Global existence, uniqueness and asymptotic stability of classical smooth solutions in one-dimensional non-linear thermoelasticity', Arch. Rational Mech. Anal. 76 (1981), 97-133.

[10] H. Triebel, 'Spaces of distributions with weights. Multipliers in $L_{p}$-spaces with weights', Math. Nachr. 78 (1977), 339-355.

[11] H. Triebel, Theory of Function Spaces (Birkhäuser-Verlag, Basel, 1983).

[12] M. Tsutsumi, 'Weighted Sobolev spaces and rapidly decreasing solutions of some nonlinear dispersive wave equations', J. Differential Equations 42 (1981), 260-281.

[13] A.I. Vol'pert and S.I. Hudjaev, 'On the Cauchy problem for composite systems of nonlinear differential equations', Math. USSR-Sb 16 (1972), 517-544.

[14] S. Zheng and W. Shen, 'Initial boundary value problems for quasilinear hyperbolic-parabolic coupled systems in higher dimensional spaces', Chinese Ann. Math. Ser. B 4 (1983), 443-462.

Institut für Angewandte Mathematik

Wegelerstrasse 10

D-5300 Bonn 1

West Germany

\author{
Department of Mathematics \\ Xi'an Jiaotong University \\ Xi'an, Shaanxi Province \\ Peoples Republic of China
}

